\title{
Terapia de presión negativa como tratamiento alternativo en el manejo de una úlcera vascular arterial de miembros inferiores
}

\author{
Catalina Quilindo Giraldoa, Julián Ramírez Román ${ }^{\mathrm{b}}$, Juan Pablo Martínez \\ a'Médica y Ciruiana. Universidad del Cauca. catalinaqg1420@gmail.com \\ (iD https://orcid.org/0000-0002-7254-7983

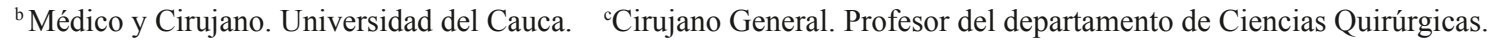 \\ Universidad del Cauca. Q.E.P.D
}

DOI 10.22517/25395203.21271

\section{Resumen}

Objetivo: Reportar un caso sobre el uso de la terapia de presión negativa como tratamiento alternativo en el manejo de una úlcera vascular arterial de miembros inferiores.

Métodos: Por medio de la historia clínica, se revisó los registros previos de una paciente en un centro de atención médica de tercer nivel en Popayán, Colombia. Posteriormente, se realizó una revisión sistemática bibliográfica a través de las bases de datos de Pubmed, ScienceDirect y Scielo.

Resultados: La terapia de presión negativa favoreció el proceso de cicatrización, permitiendo el cierre de la herida.

Conclusiones: Actualmente, este sistema se considera un apoyo fundamental en el tratamiento de las heridas complejas, las cuales suponen un reto terapéutico importante.

Palabras clave: Úlcera, proliferación celular, cicatrización, presión negativa.
Negative- pressure therapy as an alternative treatment for managing arterial vascular ulcer of the lower limbs.

\begin{abstract}
Objective: To report a case about the use of the Negativepressure wound therapy as an alternative treatment for arterial vascular ulcers presented in the lower limbs.
\end{abstract}

Methods: The patient's records were reviewed based on her medical history in a tertiary referral care center in Popayan, Colombia. Subsequently, a systematic literature review was conducted through the Pubmed database, ScienceDirect, and Scielo.

Results: The use of Negative-pressure wound therapy favored the lesion healing process.

Conclusions: Nowadays, this system is considered as an essential treatment for complex wounds, which represent a major therapeutic challenge.

Key words: Ulcers, cell proliferation, cicatrization, negative pressure.

\section{Introducción}

Desde el punto de vista demográfico, las úlceras vasculares de miembros inferiores constituyen un problema del adulto mayor y afectan principalmente a pacientes mayores de 65 años ${ }_{(1)}$. La úlcera es el deterioro de la solución de continuidad con pérdida de sustancia y epitelio producida por un proceso patológico de origen vascular. Tiene una evolución crónica y una escasa o nula tendencia a la cicatrización espontánea ${ }_{(2) \text {. Las }}$ úlceras pueden ser venosas, arteriales o mixtas. Las úlceras venosas son el tipo más común de la herida a nivel mundial y representa alrededor del $80 \%$ de todas las úlceras de las piernas que afectan principalmente a personas mayores de 65 años de edad ${ }_{(3)}$. Las úlceras arteriales son alrededor del 10\% al 15\% de los casos y están producidas por un déficit de riego sanguíneo, siendo la obstrucción aterosclerótica la causa más importante. En su tratamiento, se utilizan desbridamientos quirúrgicos, medidas posturales o transferencias de tejidos ${ }_{(5)}$. Por otro lado, la terapia de presión negativa o terapia VacuumAssistedClosure (VAC) ha mostrado ser útil en el manejo de heridas complejas como abdomen abierto, heridas infectadas y úlceras venosas ${ }_{(6)}$. Sin embargo, su utilidad en las úlceras arteriales todavía no se ha determinado. En este trabajo se describe el caso de una paciente ulcerosa manejada con terapia de presión negativa. 


\section{Reporte de caso}

Se trata de una paciente de sexo femenino de 65 años de edad, casada, procedente de la zona rural del Departamento del Cauca, Colombia, con antecedentes médicos de hipertensión arterial, taquicardia supra ventricular paroxística y enfermedad arterial oclusiva crónica de miembros inferiores; con previo reporte angiográfico de enfermedad severa intrapoplitea con oclusión total y subtotal de la arteria tibial anterior y tibial posterior con discreta canulación distal por circulación colateral y peroneas filiformes, con predominio de miembro inferior derecho. Ulteriormente, se efectuó una angioplastia con revascularización de la arteria tibial anterior derecha.

La paciente consultó en un primer nivel de atención por úlcera que comprometía el cuarto y quinto artejo del pie derecho y hallux del pie izquierdo. Inicialmente, fue valorada en dicho centro de salud y posteriormente remitida a una institución de tercer nivel. Al ingreso, se encontraba estable, con presión arterial de $130 / 80 \mathrm{mmHg}$, frecuencia cardiaca de 85 por minuto, frecuencia respiratoria de 16 por minuto y temperatura de $36,7^{\circ} \mathrm{C}$. Además, refería dolor intenso en el hallux izquierdo.

En el examen físico se observaba levantamiento de uña, secreción purulenta y fétida. Conjuntamente se evidenció necrosis en el cuarto y quinto artejo del pie derecho y en el hallux del pie izquierdo, por lo que se propuso amputación de los mismos.

Se decidió llevar a cirugía, donde se realizó desarticulación del hallux izquierdo, con cierre primario de la herida, resección de la cápsula articular y osteotomía distal del primer metatarsiano; desarticulación del cuarto y quinto artejo del pie derecho, osteotomía del cuarto y quinto metatarsiano hasta el tercio proximal y desbridamiento de la úlcera dorsal del pie derecho de aproximadamente $7 \times 5 \mathrm{~cm}$, con tejido necrótico y tendones desvitalizados sin protección (Figura 1).
Figura 1. Aspecto de úlcera después de la amputación del 4 y 5 artejo y desbridamiento quirúrgico.

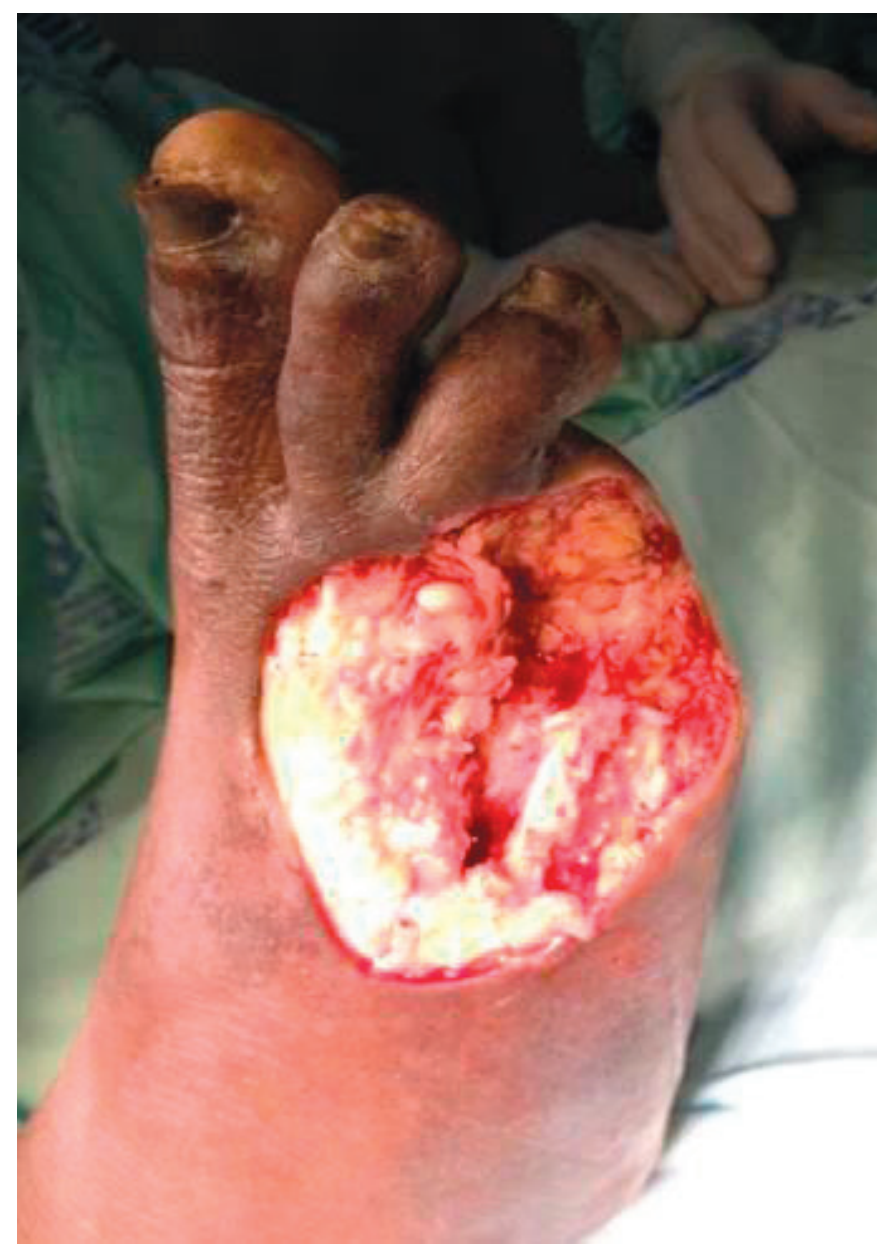

Eldesbridamiento quirúrgico se llevó a cabo sin complicaciones, con aplicación funcional del sistema VAC en el pie derecho, que consta de una esponja de poliuretano que se acopla a un tubo de drenaje no colapsable, y éste, a su vez, a una bomba de aspiración, con el fin de garantizar la distribución uniforme de la presión en toda la herida.

Luego del desbridamiento se recortó la esponja a la medida de la lesión y se colocó el apósito transparente sobre el tubo de drenaje y la esponja, tomando la precaución de que el tubo de aspiración no abarcara la piel sana, ya que podía causar más lesión y pérdida del tejido sano. El sistema fue programado en terapia intermitente con presión de $125 \mathrm{mmHg}$ e intensidad 30 mmHg (Figura 2). 


\section{Figura 2. Presión negativa}

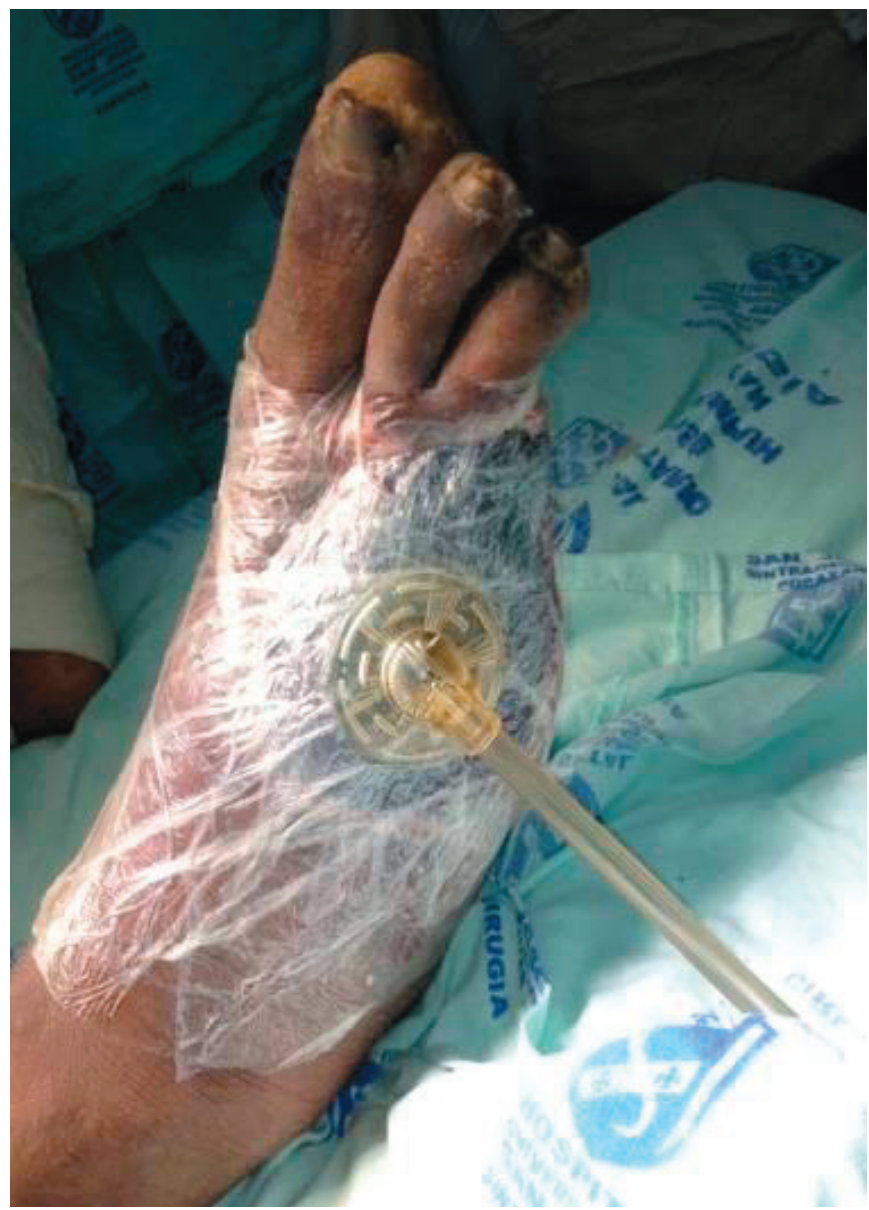

Durante la hospitalización, la paciente requirió dos recambios del sistema VAC con una duración de ocho días cada uno, los cuales fueron programados en terapia intermitente con la misma presión e intensidad del primer recambio. Además, la paciente recibió manejo por comité de curaciones durante 12 días, de manera continua y progresiva (Figura 3 ).

\section{Figura 3. Resultado final}

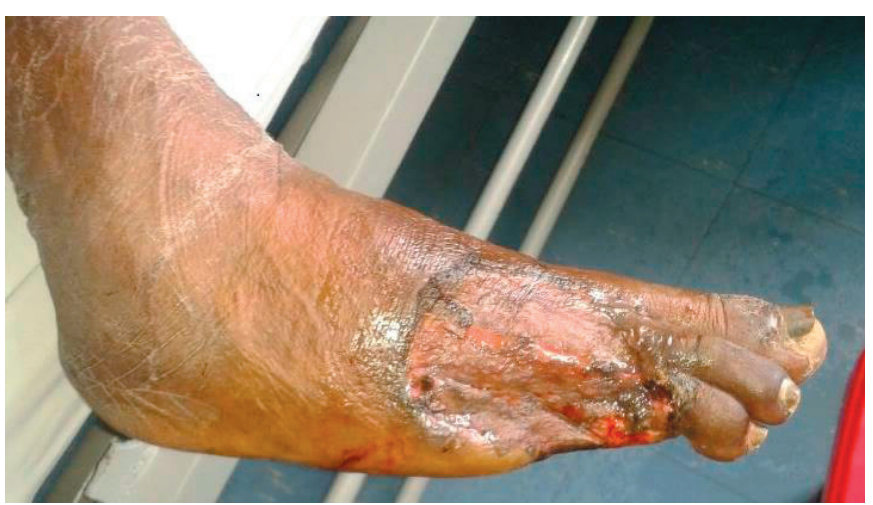

Finalmente, la paciente fue valorada por cirugía plástica, que realizó cobertura de la lesión ulcerosa mediante injertos cutáneos de espesor parcial y seis días después se determinó la salida de la paciente. El tiempo total de hospitalización total fue de 28 días.

Cabe señalar que la paciente autorizó el procedimiento y se obtuvo su consentimiento informado, así como el de sus familiares, tanto para el tratamiento como para la aplicación de las esponjas con el sistema de vacío. Además, aceptando que este reporte de caso se realizaría con fines académicos sin conflictos de interés, conservando los criterios de confidencialidad y privacidad.

\section{Discusión}

La insuficiencia arterial periférica es un conjunto de cuadros sindrómicos, agudos o crónicos, generalmente derivados de la presencia de una enfermedad arterial oclusiva que condicionan un insuficiente flujo sanguíneo a las extremidades ${ }_{(7)}$. Los pacientes con esta enfermedad tienen posibilidades de desarrollar lesiones tróficas en las extremidades, tales como ulceras isquémicas o gangrena ${ }_{(8)}$.

El tratamiento endovascular representa una alternativa atractiva para pacientes con isquemia crítica de miembros inferiores al reducir el riesgo de amputación puesto que es capaz de lograr el rescate del miembro isquémico a corto o largo plazo en algunos casos ${ }_{(9)}$.Sin embargo, la paciente descrita recibió este tratamiento no siendo favorable para su padecimiento crónico de base. Al llegar con esta enfermedad, a la paciente se le realizó el desbridamiento oportuno para disminuir más su gravedad. Entre las opciones del tratamiento está la cobertura de herida, la cual debe hacerse lo más eficientemente posible. Una vez que esté limpia y bien vascularizada se puede escoger entre diferentes opciones, ya sea por intención secundaria o por injerto de piel ${ }_{(10)}$.

No siempre es necesaria la sedación, que depende del estadio previo de salud del paciente, la profundidad y ubicación de la herida y la experiencia del cirujano ${ }_{(11)}$. En los últimos años se agregó a las modalidades para el cierre de heridas agudas y crónicas $_{(12)}$, múltiples dispositivos de terapia de presión negativa presentes hoy en el mercado, entre ellos se encuentra el sistema VAC, utilizado en este caso para el manejo de una herida crónica de miembros inferiores secundario a la insuficiencia arterial periférica.

La aplicación de presión negativa con fines terapéuticos se remonta al año 1841 en que Junod aplicaba campanas de vidrio que provocaban succión, sobre la piel sana, generando lo que él denominaba "hiperemia terapéutica" ${ }_{(13)}$. En 1952, Raffel describió la aplicación de drenajes utilizando presión negativa bajo colgajos dermograsos, luego de una mastectomía, con el 
fin de evitar complicaciones postoperatorias como seroma, hematoma e infección ${ }_{(14)}$. Luego de varios refinamientos, es una técnica que hoy en día continúa siendo utilizada.En 1966, varios autores rusos como Davydov, Iankov, Inoiatov y Kochev aplicaron este concepto de colocación de drenaje aspirativo en heridas cerradas ${ }_{(14)}$.

En 1989, en los Estados Unidos, Zamierowski, un cirujano plástico, patenta un dispositivo que permite la irrigación y evacuación continua de heridas cubierto por una membrana impermeable ${ }_{(9)}$. El mismo método fue descrito por Fleischmann en 1993, en Alemania, aplicando presión subatmosférica en 15 pacientes con fracturas expuestas. Los autores reportaron que los resultados del tratamiento fueron una "eficaz limpieza y acondicionamiento de las heridas, con una marcada proliferación de tejido de granulación" ${ }_{(15)}$ sin infecciones óseas. Posteriormente, los mismos autores lo utilizaron para el manejo del síndrome compartimental en miembros inferiores y de infecciones agudas y crónicas con buenos resultados ${ }_{(15)}$.

Desde su descripción inicial, varios autores han sugerido que el mecanismo de acción radica en promover la curación de las heridas mediante la aplicación de presión negativa, de manera que el uso de niveles controlados de presión subatmosférica y succión controlada, aceleren la resolución de las heridas, favoreciendo la granulación ${ }_{(5)}$, reduciendo el edema tisular, estimulando la proliferación de tejidos adyacentes a la herida y disminuyendo los niveles bacterianos ${ }_{(11)}$. Tales efectos se evidenciaron en la paciente durante el trascurso del tiempo, generando menor proceso inflamatorio y promoviendo la formación de tejido de granulación para una posterior cirugía.

La aplicación de presión negativa disminuye la colonización bacteriana de heridas infectadas ${ }_{(16)}$, favorece la contracción de la herida, aumento en la proliferación celular y la síntesis protéica, al modificarse el cito esqueleto celular; fenómeno que sucede por la alteración en los puentes de integrinas intercelulares causada por las fuerzas mecánicas de estiramiento que se producen por la presión negativa que sufre la herida ${ }_{(17)}$. También provee un ambiente húmedo propicio para la cicatrización, protege de contaminación externa y puede permitir la reducción de medicamentos contra el dolor (17). Al mismo tiempo, la remoción del exudado crónico de la herida, es un proceso beneficioso, ya que remueve métalo proteinasas o factores inhibitorios de la cicatrización (18), características que se hicieron evidentes en el resultado final de la paciente.

La presión negativa se puede regular en ciclos continuos o intermitentes y en un ámbito de presión que se ajuste al tipo y características de la lesión ${ }_{(17)}$. En la práctica clínica, la presión mínima de inicio es de $125 \mathrm{mmHg}$ e intensidad de $30 \mathrm{mmHg}$, de forma intermitente, con succión continua por cinco minutos y posteriormente dos minutos sin succión ${ }_{(19)}$. Este tipo de presión e intensidad se utilizó en la paciente con succión continua y controlada, en los tipos de recambio del sistema VAC.

Las heridas tratadas con la técnica VAC pueden ser agrupados en nueve categorías descriptivas: 1) aquellas a las que se aplica un injerto de piel de espesor parcial; 2) infectadas (después del desbridamiento); 3) de fractura abierta; 4) de tejidos blandos agudas (con exposición del tendón, los huesos y/o articulaciones); 5) defasciotomía después del síndrome compartimental; 6) crónicas (duración mayor de tres meses), 7) las quirúrgicas que son difíciles de cerrar debido a la tensión; 8) con pines externos de fijación o tubos o en los sitios de catéter con irritación y drenaje y 9) heridas quirúrgicas que producen líquido seroso después del día del postoperatorio segundo ${ }_{(20)}$.

Existen pocos casos publicados sobre el sistema VAC en tratamiento de úlceras por enfermedad arterial oclusiva crónica. Jiménez reporta dos casos sobre el manejo de úlceras vasculares arteriales, en los que se lograron buenos resultados, al igual que en el caso expuesto ${ }_{(4)}$. Sin embargo, la evaluación clínica de la cicatrización de heridas es subjetiva ${ }_{(9)}$. Esto hace que sea difícil de producir una investigación clínica sólida que garantice claramente la eficacia de las técnicas de VAC o de otro tipo en comparación con las alternativas ${ }_{(20)}$. Es primordial que la causa de base de la úlcera se controle, es decir, que se obtenga un buen flujo sanguíneo ${ }_{(4)}$, ya que el uso de la terapia de presión negativa es fundamental para favorecer exclusivamente el manejo local de la herida, más no el origen de la misma.

\section{Conclusión}

Se presenta un caso clínico de terapia de presión negativa como tratamiento de úlcera vascular arterial causado por la enfermedad arterial oclusiva grave a nivel de los miembros inferiores que fue manejada con este sistema en pie derecho con una respuesta satisfactoria.

La aplicación del sistema VAC con fines terapéuticos en el caso clínico documentado revela que los resultados del tratamiento fueron eficaces, con lo cual favoreció el cierre por segunda intención y por consiguiente redujo la duración del tiempo hospitalario (4). Sin embargo, la terapia de presión negativa en el tratamiento de úlceras por enfermedad vascular no está definido aún debido a la escasez de información y los poco casos reportados sobre su uso, siendo necesario realizar nuevas investigaciones que evalúe su efectividad. 


\section{Referencias}

1. Otero González G, Agorio Norstrom C, Martínez Asuaga, M. Úlceras de miembros inferiores: características clínicoepidemiológicas de los pacientes asistidos en la unidad de heridas crónicas del Hospital de Clínicas. Revista Médica del Uruguay. 2012; 28(3):p. 182-189.

2. Tejada Osegui, A. Tratamiento de las úlceras vasculares mediante la terapia de presión negativa 2014.

3. Salomé GM, de Almeida SA, Ferreira L M. Evaluation of pain in patients with venous ulcers after skin grafting. Journal of Tissue Viability. 2014; 23(3):p. 115-120.

4. Jimenez CEJ. Terapia de presión negativa: una nueva modalidad terapéutica en el manejo de heridas complejas, experiencia clínica con 87 casos y revisión de la literatura. Revista Colombiana de Cirugía. 2007; 22(4): p.209-224.

5. Buendía Pérez J, Vila Sobral A, Gómez Ruiz R, Qiu Shao SS, Marré Medina D, Romeo M, et al. Tratamiento de heridas complejas con terapia de presión negativa: Experiencia en los últimos 6 años en la Clínica Universitaria de Navarra, Pamplona (España). Cirugía Plástica Ibero-Latinoamericana. 2011; 37, S65-S71.

6. Trujillo-Martín M, García-Pérez, L, Serrano-Aguilar, P. Efectividad, seguridad y coste-efectividad de la terapia por presión negativa tópica para el tratamiento de las heridas crónicas: una revisión sistemática. Medicina clínica. 2011; 137(7): p. 321-328.

7.Hernando FJS, Conejero AM. Enfermedad arterial periférica: aspectos fisiopatológicos, clínicos y terapéuticos. Revista española de cardiología. 2007; 60(9): p. 969-982.

8. García F A, García JRM, de Haro Miralles J, Casariego CV. Síndrome de isquemia crónica de las extremidades inferiores: definición, etiología, clínica, diagnóstico y posibilidades terapéuticas. Medicine: Programa de Formación Médica Continuada Acreditado. 2009; 10(45):p. 2994-3007.

9. Merchán-Galvis ÁM, Concha-Sandoval JM. Sistema de presión negativa en el tratamiento de las fracturas abiertas: Presentación de caso clínico y revisión de la literatura. Revista de la Facultad de Ciencias de la Salud de la Universidad del Cauca. 2013; 15(2):p. 21-25.

10. Brian MP, Julian JP. Reconstrucción de extremidad inferior. Revista Médica Clínica Las Condes. 2010; 21(1): p. 76-85.
11. Macedo FB, Carriquiry CE. Tratamiento de heridas utilizando presión negativa tópica. Biomedicina. 2006; 2(2): p. 122-130.

12. González JMD, Llorente RAN, Cherit JD, Ruiz JC. Relevancia del tratamiento integral del paciente con heridas extensas previo a la terapia con presión negativa. Dermatología Revista Mexicana. 2008; 52(4):p. 182-187.

13. Greer SE.Whither subatmospheric pressure dressing?. Annals of plastic surgery. 200; 45(3):p. 332-334.

14. Blackburn. Negative pressure dressings as a bolster for skin grafts. Annals of Plastic Surgery. 1998; 40(3):p. 453-7.

15. Thomas, S. An introduction to the use of vacuum assisted closure. World wide wounds.2001; 9.

16. Morykwas MJ, Argenta LC, Shelton-Brown EWM. Vacuum-assisted closure: a new method for wound control and treatment: animal studies and basic foundation. 1997;38(6): p.553-62.

17. Elizondo Almeida J, Pucci Coronado J, Soto-Pacheco L. Cierre Asistido con Presión Negativa (VAC), en el tratamiento de esternotomía infectada: primer caso en Latinoamérica. Revista Costarricense de Ciencias Médicas. 2001; 22(1-2): p.59-64.

18. MagedS.S. Saleh, Al-amin MSAMA. Negative Pressure Wound Therapy for Treatment of Extensive Degloving Injury of the Left Lower Limb Associated with Open Fracture of the Femur (Case Report) Med. J. Cairo Univ. 2012; 80:p.361-5.

19. Hernández JMR, Pérez MB, Medina RS, Espriella CM, Trejo AT, Chew CL. Negative pressure system in the sepsis management by open abdomen. Medicina Crítica. 2007; 21(2):p.74-79.

20. Archdeacon M T, Messerschmitt P. Modern papineau technique with vacuum-assisted closure. Journal of orthopaedic trauma. 2006; 20(2):p.134-137.

21. Herranz ML. Prevalencia de úlceras vasculares de extremidad inferior. Revisión sistemática. Diseño de una guía terapeútica basada en criterios etiopatogénicos y anatomoclínicos. REDUCA (Enfermería, Fisioterapia y Podología). 2011; 3(2). 